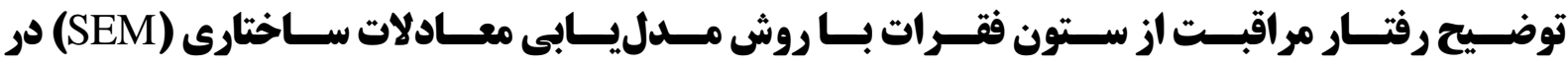 دانش آموزان: كاربرد نظريئ شناختى اجتماعى
}

\author{
زهرا اكبرى جهره برق'؛ صديقه سادات طوافيان "، على منتظرى \\ 1. I دانشكده علوم بزشكى، دانشكاه تربيت مدرس، تهران، ايران

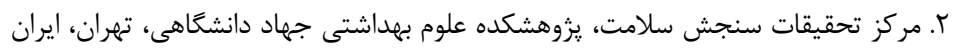

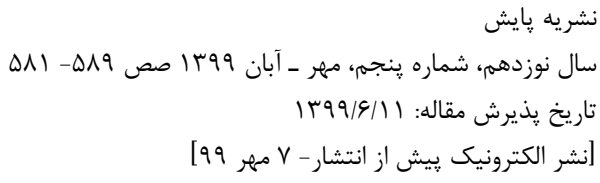

جكيده

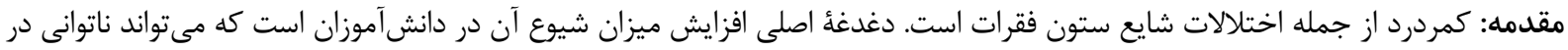

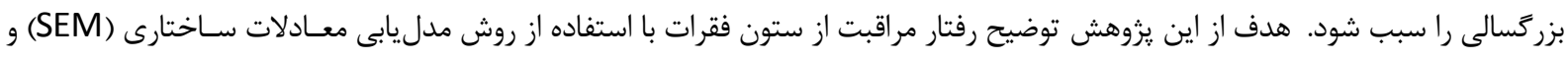

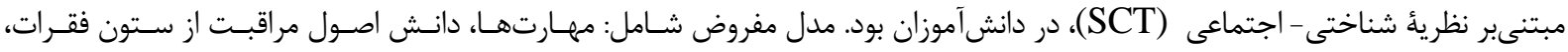

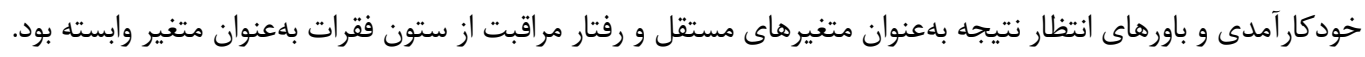

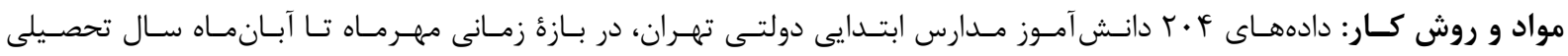

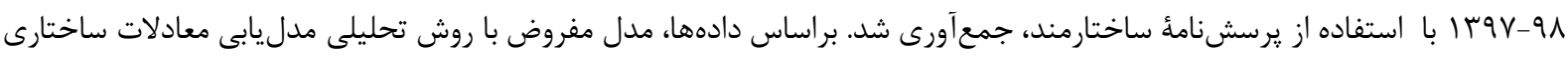

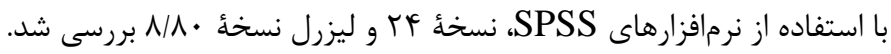

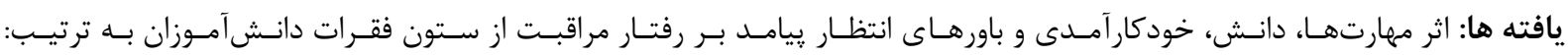

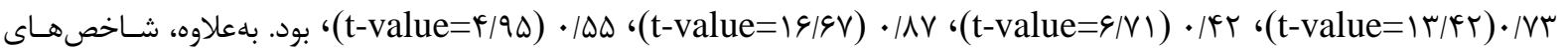

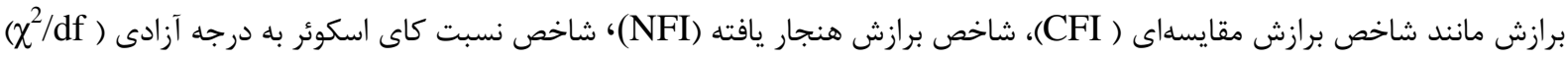

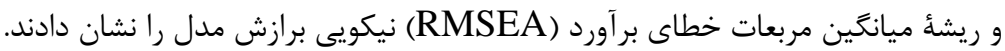

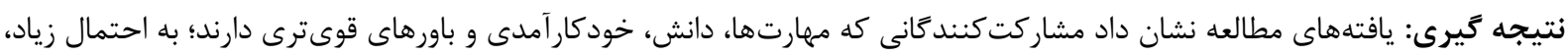

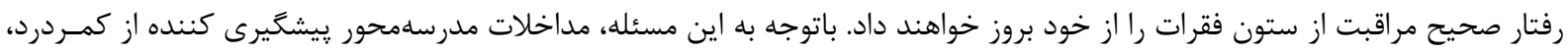

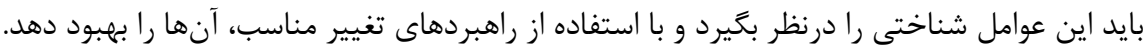

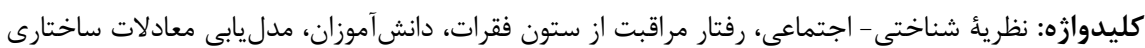

كد اخلاق: IR.TMU.REC.1396.727 
فرد هستند. بهعبارت سادهتر، اين عوامل مهم فردى تعيين مى كنند

كه شخصى دركير رفتارى بشود يا نشود [1 [1]]

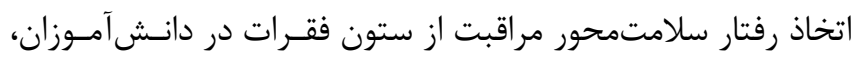

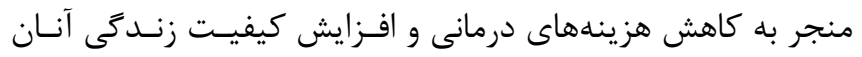

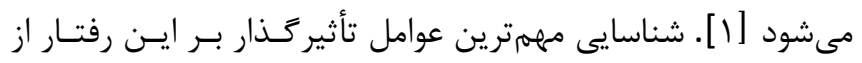

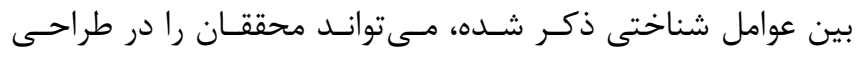

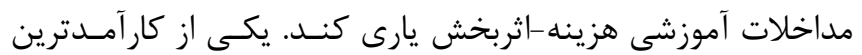

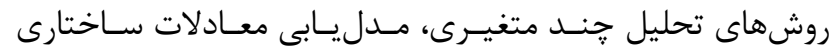

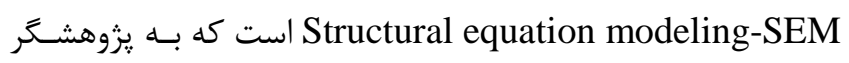

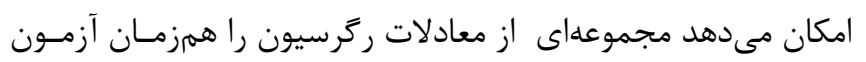

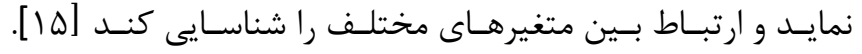

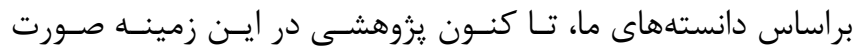

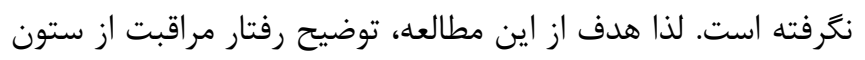

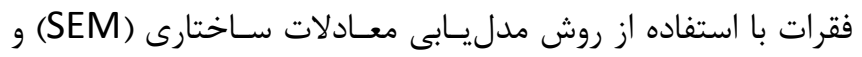

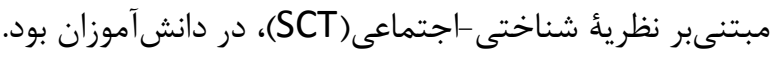

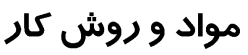

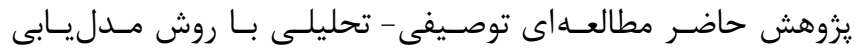

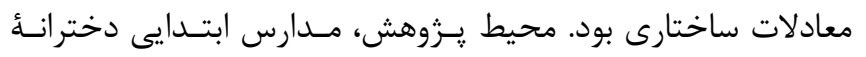

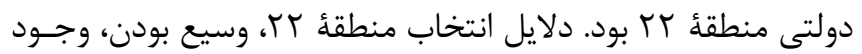

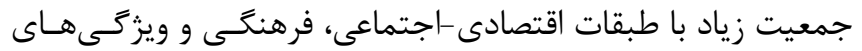

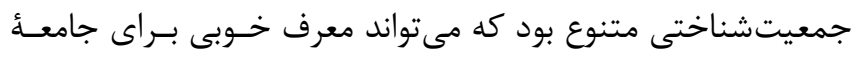

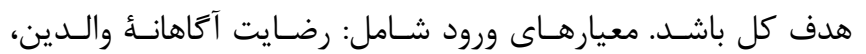

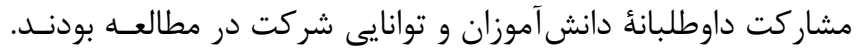

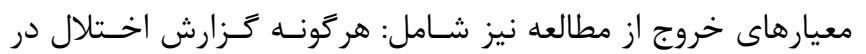

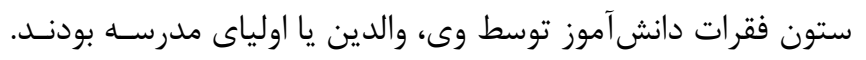

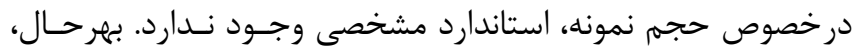

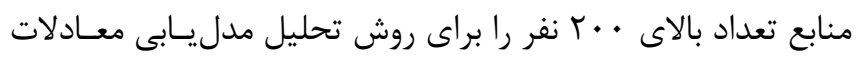

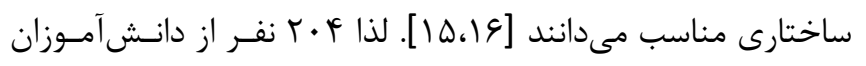

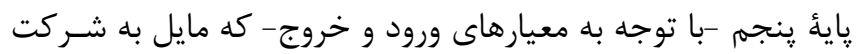

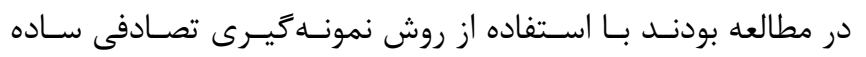

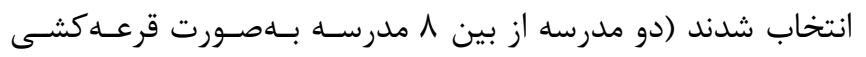

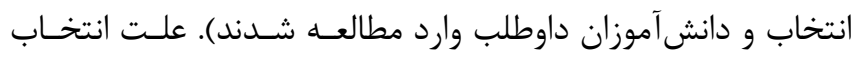

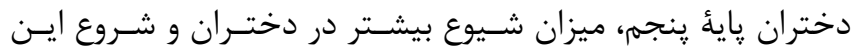

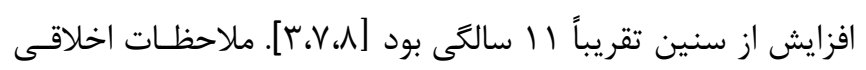

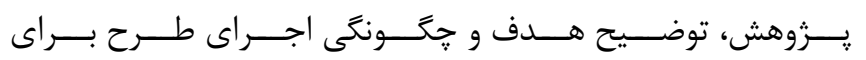

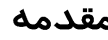

در سراسر دنيا، كمردرد بهعنوان عامل نـاتوانى بزركســالان، ازجملـهـ

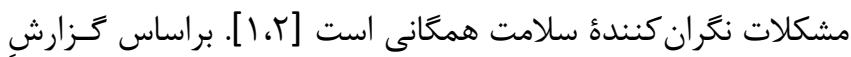

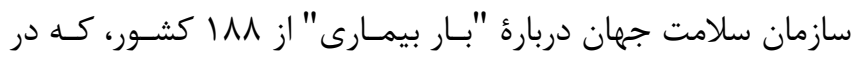

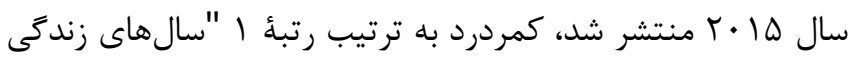

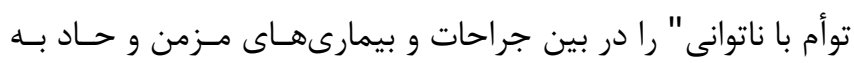

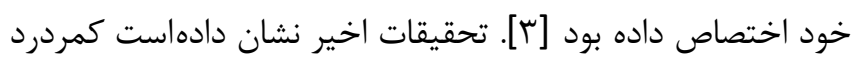

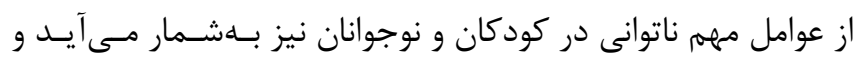

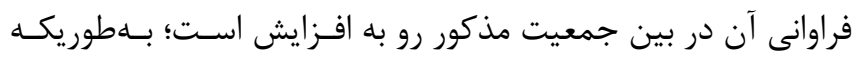

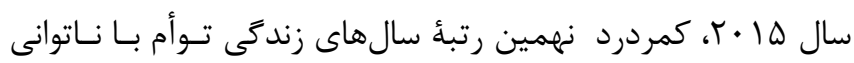

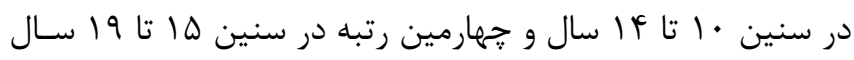

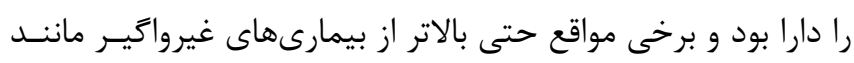

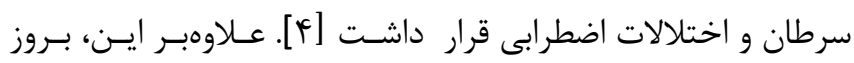

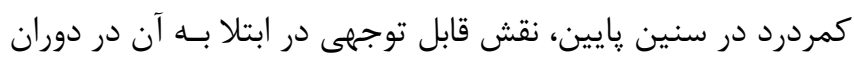

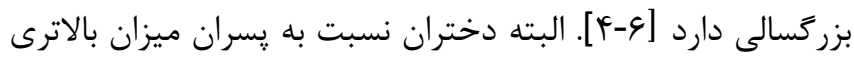

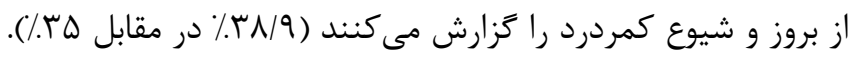

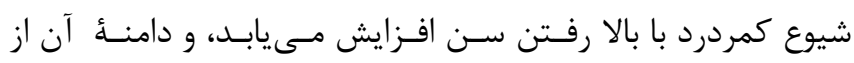

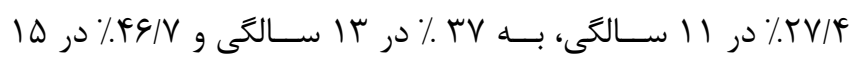

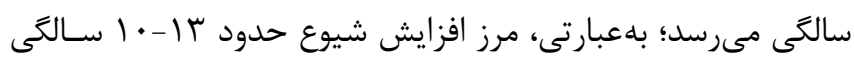

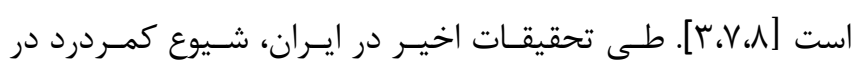

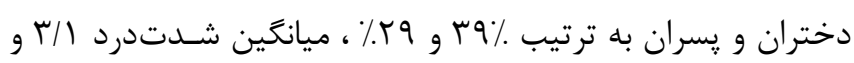

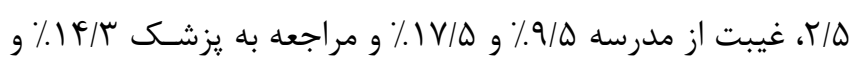

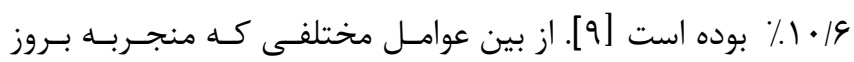

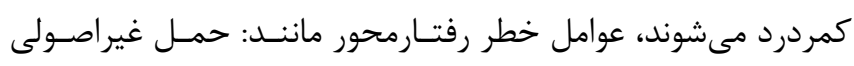

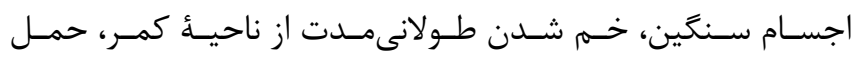

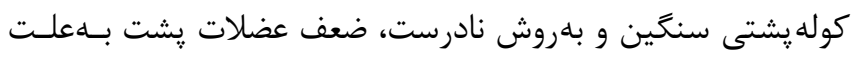

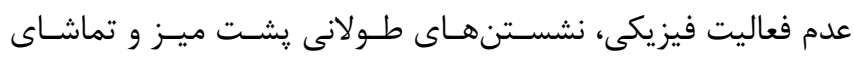

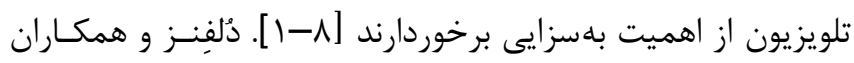

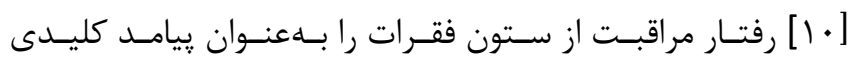

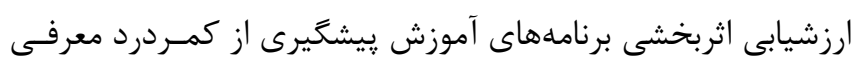

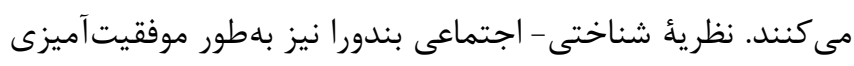

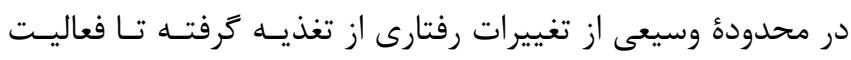

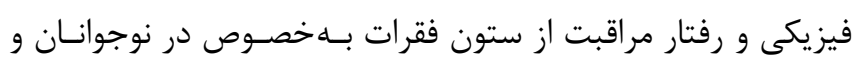

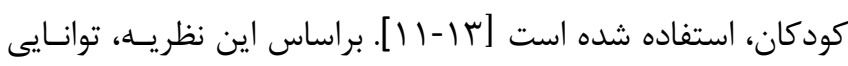

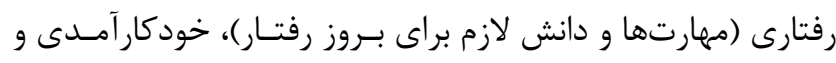

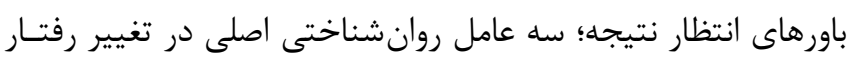


جامعهُ خاص، با استفاده از دادههاى همبستخى آزمون نمايـد. بــراى

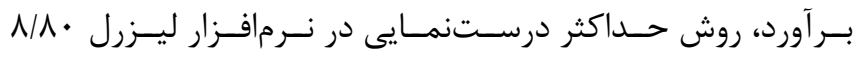

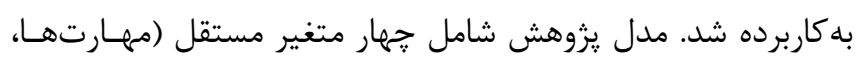

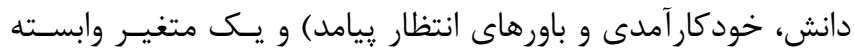

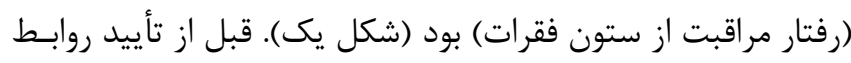

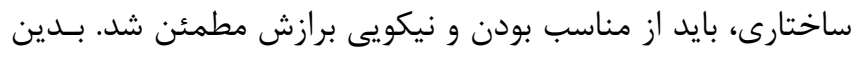

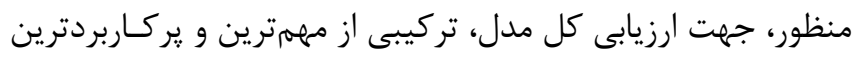

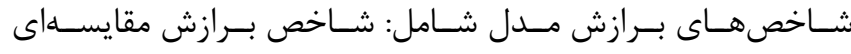
Comparative Fit Index (CFI) Normed Fit Index (NFI)

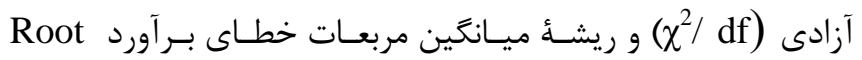
Mean Squared Error of Approximation (RMSEA)

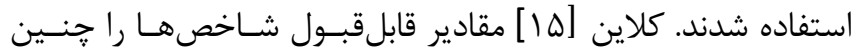

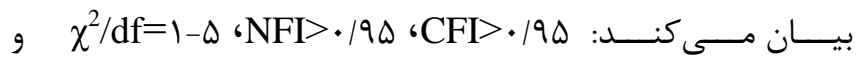

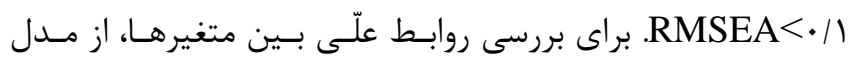

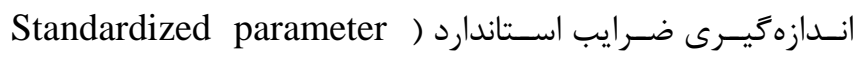

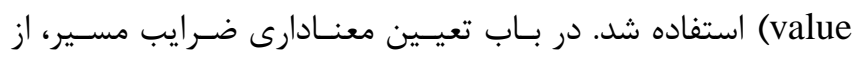

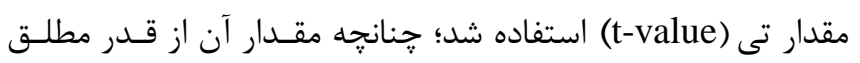

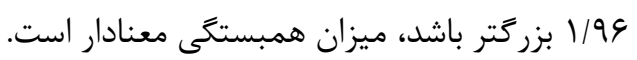

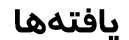

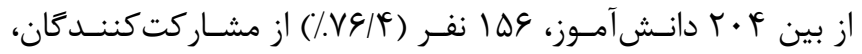

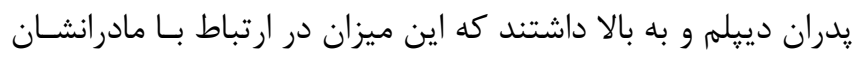

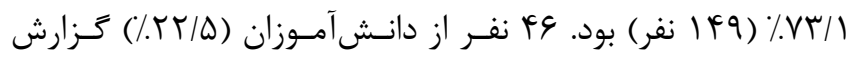

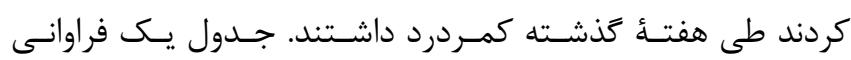

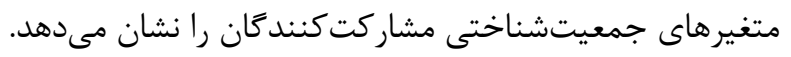

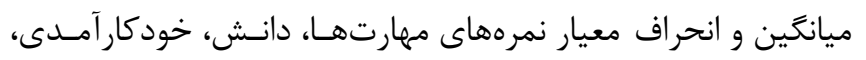

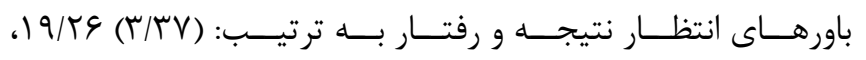

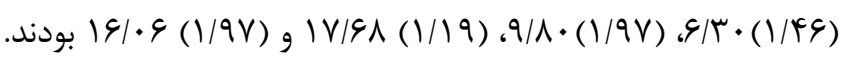

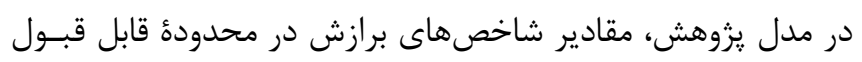

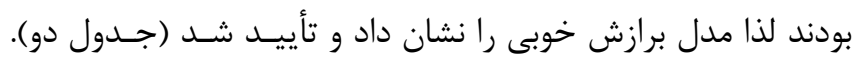

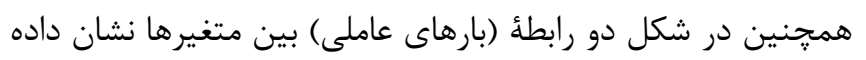

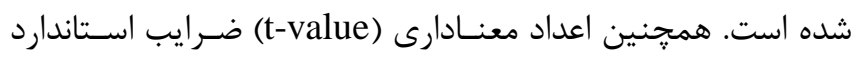

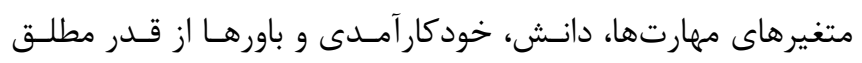

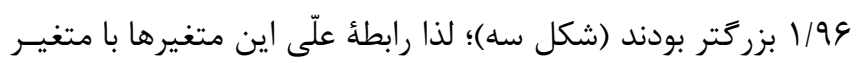
رفتار تأييد شد. برز

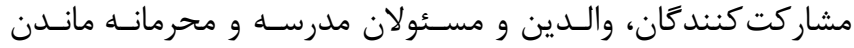

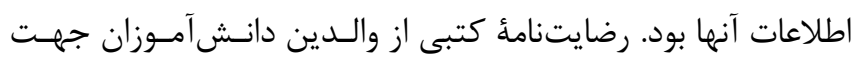
شركت در مطالعه كرفته شد.

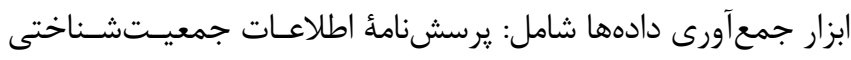

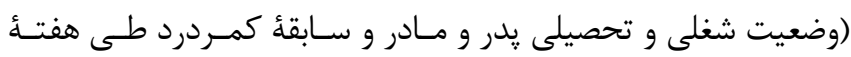

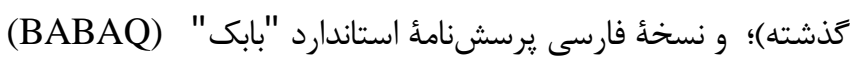

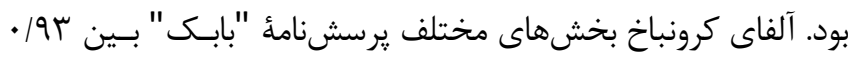

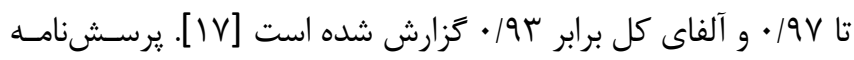
شامل ه زيرمقياس بود كه به شرح ذيل مى الشاشند:

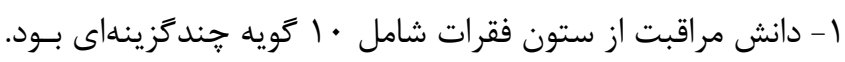

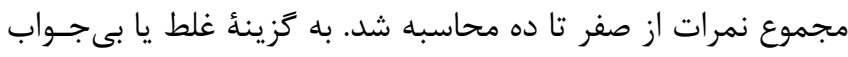

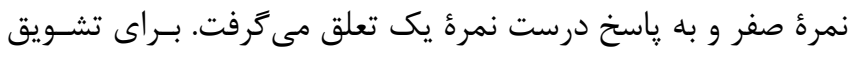

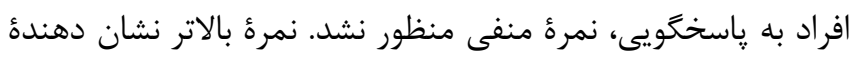
دانش بالاتر بود.

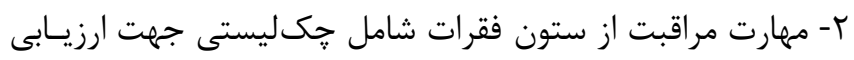

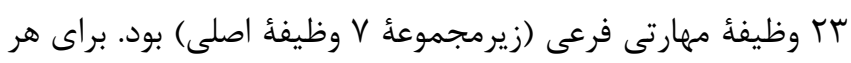

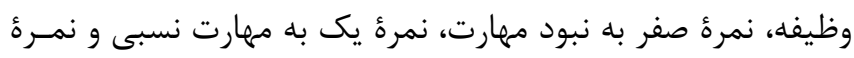

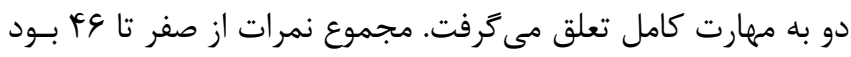

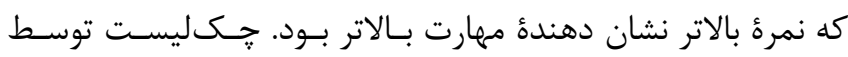

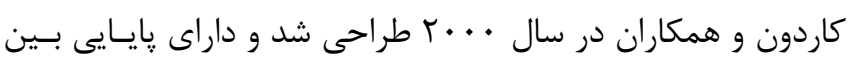
مشاهده كنند

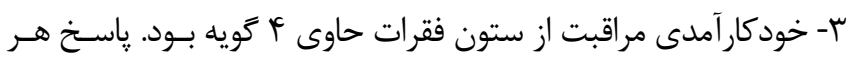

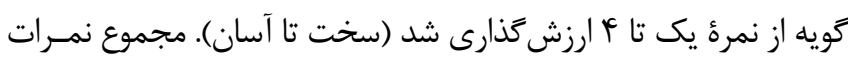

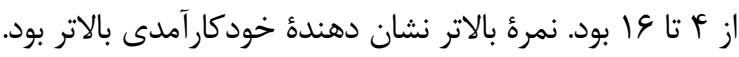

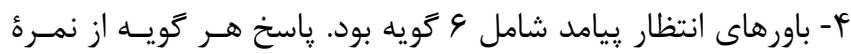

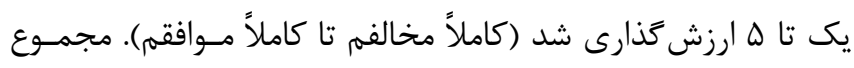

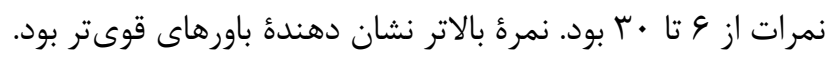

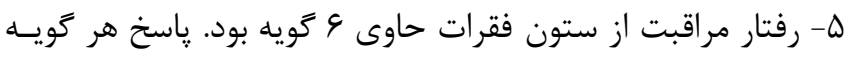

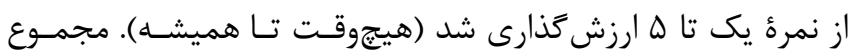

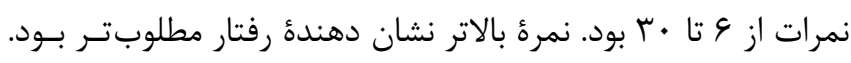

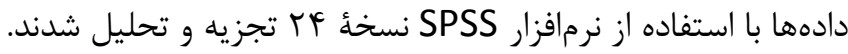

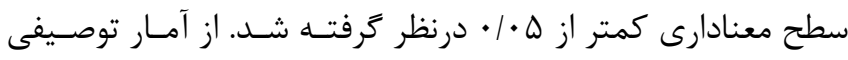

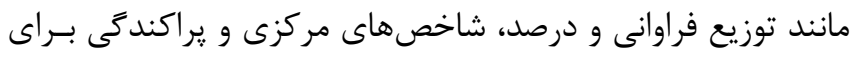

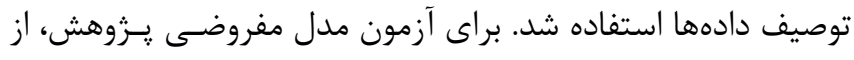

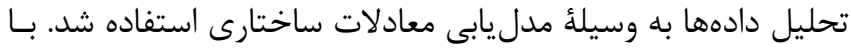

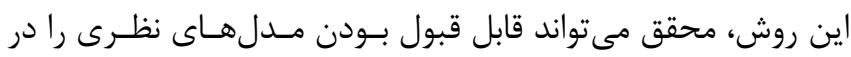


جدول ا: توزيع فراوانى متغيرهاى جمعيتشناختى مشاركت كنندكًان (تعداد F.F نفر )

\begin{tabular}{|c|c|}
\hline (درصد) فراوانى & متغيرهاى جمعيت شناختى \\
\hline & شغل بدر \\
\hline$|\Lambda|(\Lambda \Lambda / \Lambda)$ & شاغل \\
\hline$F(r)$ & بيكار \\
\hline$\|(\Delta / \uparrow)$ & بازنشسته \\
\hline & 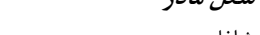 \\
\hline$f \cdot(19 / 9)$ & 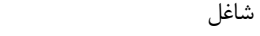 \\
\hline \multirow{2}{*}{$19 \cdot(V / / F)$} & خانهدار \\
\hline & تحصيلات بدر \\
\hline$r(9 / 9)$ & بىسواد/ابتدايى \\
\hline$\Lambda \vee(F T / \varphi)$ & دييلهم \\
\hline \multirow[t]{2}{*}{$99(\Pi r / \Lambda)$} & دانشگاهى \\
\hline & تحصيلات مادر \\
\hline rf (Ig/V) & بىسواد/ابتدايى \\
\hline $9 F(f 9 / 1)$ & دييلم \\
\hline \multirow[t]{2}{*}{$\Delta \Delta(T V)$} & دانشَاهى \\
\hline & داشتن يشتدرد \\
\hline$F \in(T Y / Q)$ & بلى ب \\
\hline $\mid \Delta F(V D / \Delta)$ & 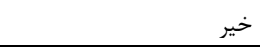 \\
\hline
\end{tabular}

جدول r: مقادير شاخصهاى نيكويى برازش مدل مفروضى

\begin{tabular}{|c|c|c|c|c|}
\hline نتيجه & CFI & RMSEA & NFI & $\chi^{2} /(\mathbf{d f})$ \\
\hline تأييد مدل &.$/ 99$ & $\cdot 1 \cdot 19$ &.$/ 9 V$ & $r|\Delta|$ \\
\hline
\end{tabular}

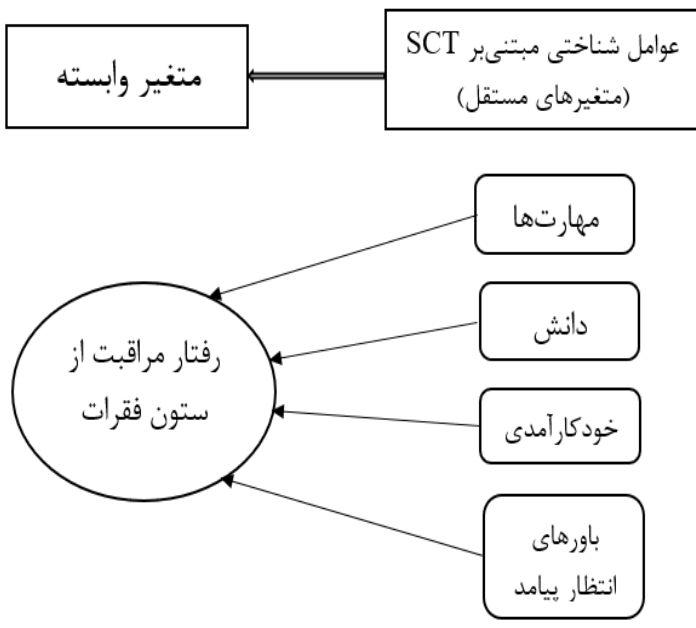

شكل ا: مدل مفروضى روابط بين متغيرهاى مستقل و وابسته 


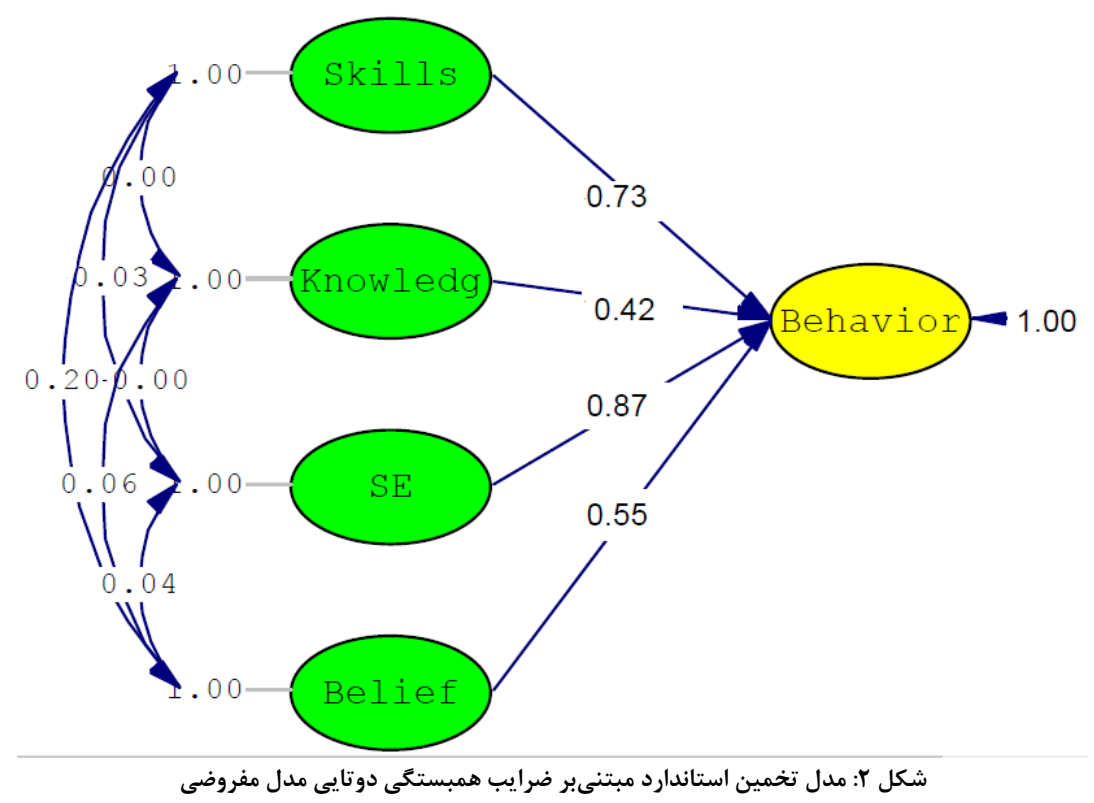

شكل r: مدل تخمين استاندارد مبتنىبر ضرايب همبستكى دوتايى مدل مفروضى

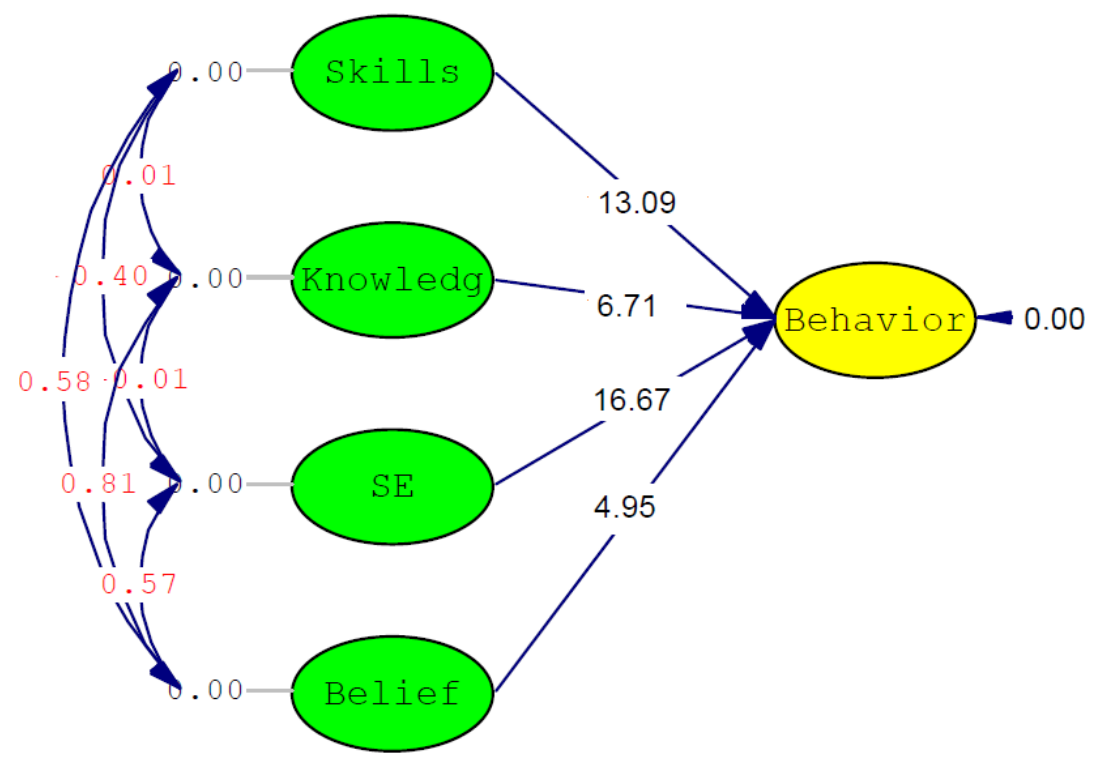

شكل r: مدل اعداد معنادارى مدل مفروضى

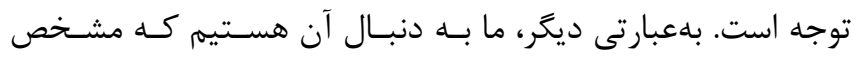

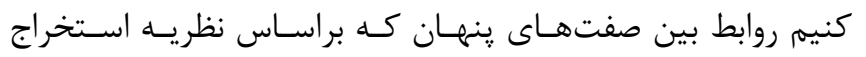

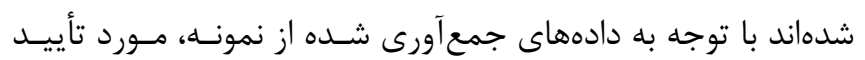

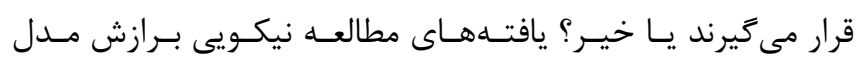

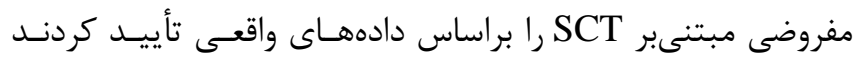

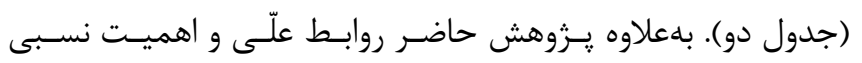

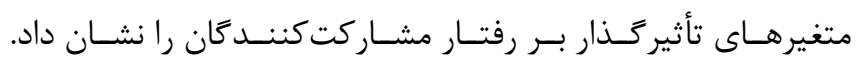

\section{بحث و نتيجه گيرى}

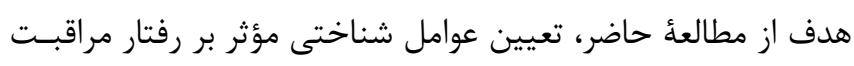

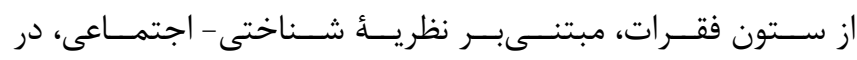

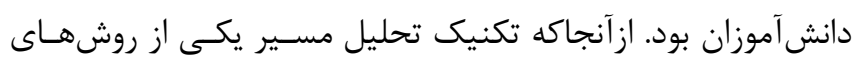

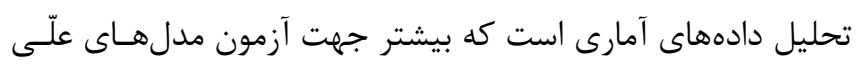

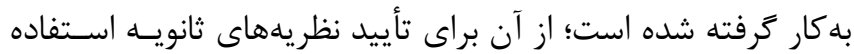

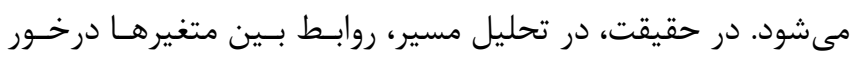


ارتباط بين متغير دانش و رفتار مراقبت از ستون فقرات در اين مدل

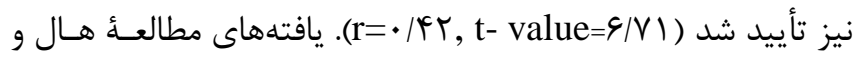

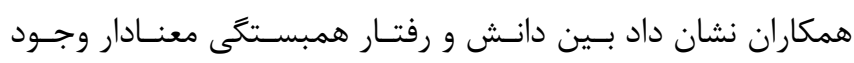

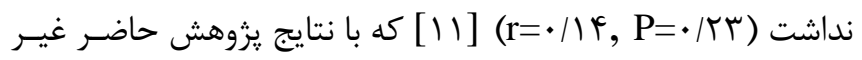

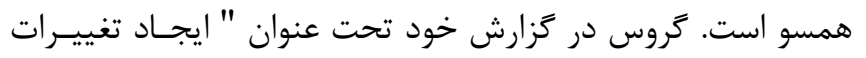

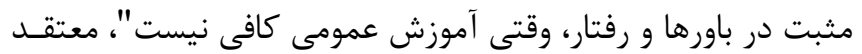

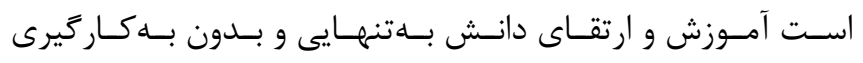

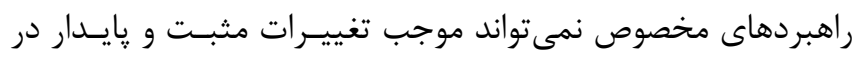

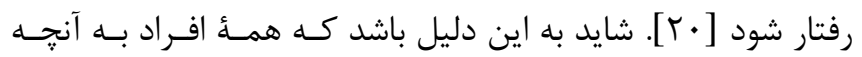

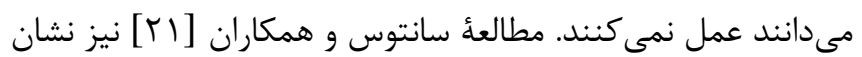

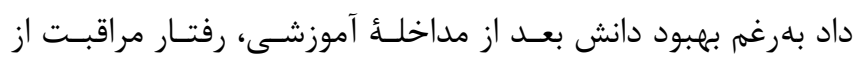

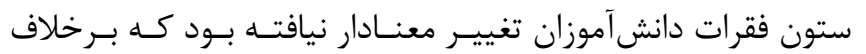
نتيجأ يزوهش حاضر است. به اعتقاد محقق، دانش نقش كليـدى در درات

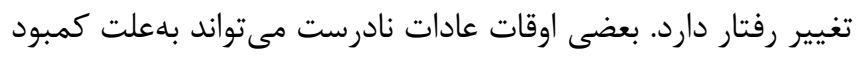

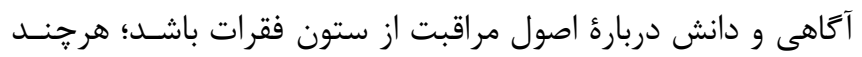

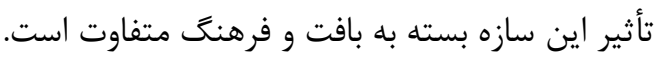

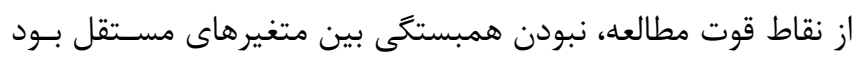

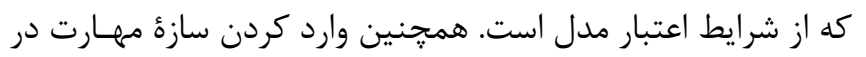

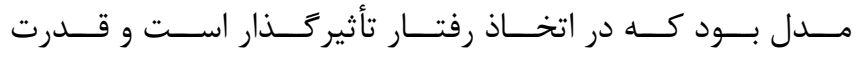

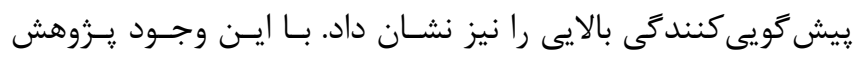

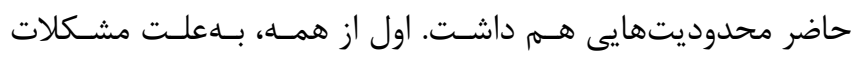

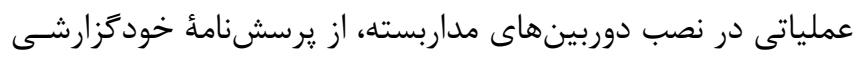

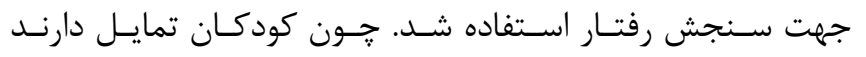

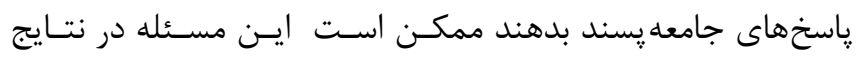

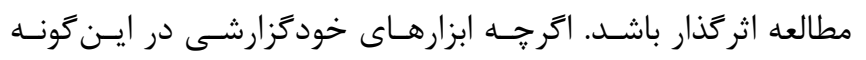

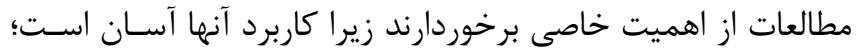

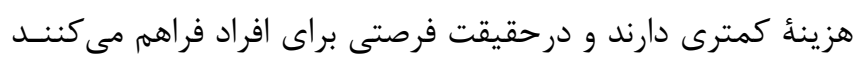

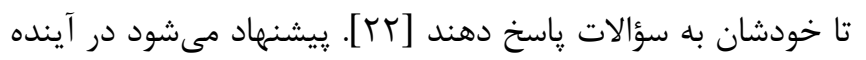

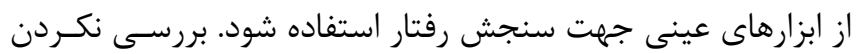

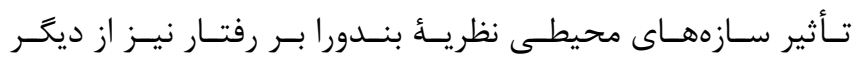

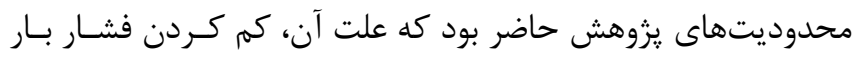

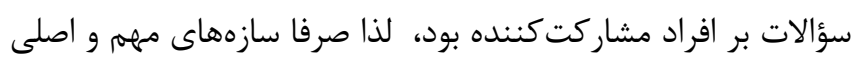

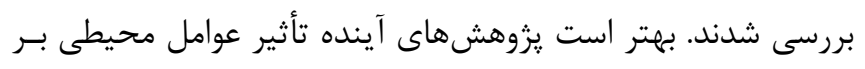

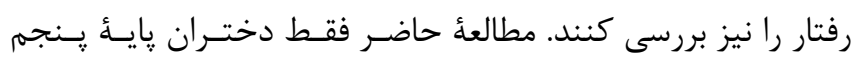

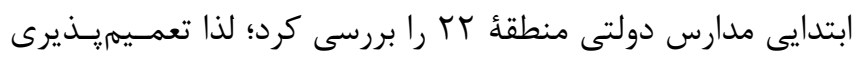

همانطور كه از شكلهاى دو و سـه قابـل اسـتنباط اسـت، تحليـل

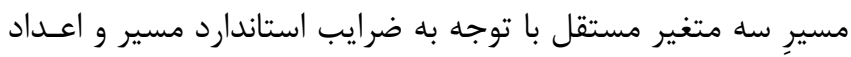

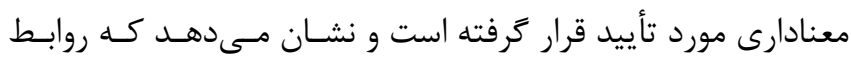

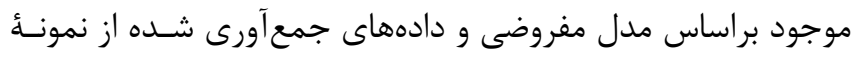

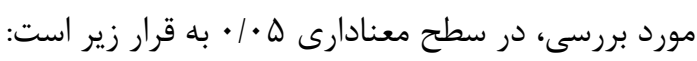

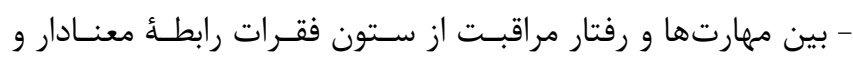
مستقيمى وجود دارد.

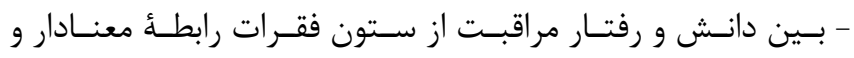

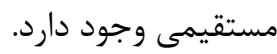
- بين خود كار آمدى و رفتار مر اقبت از ستون فقرات رابطئ معنـادار و

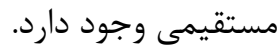

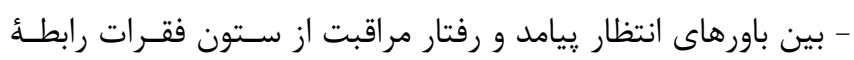

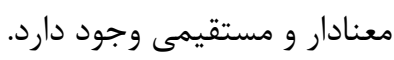

- بين متغيرهاى مستقل رابطهٔ معنادار و مستقيمى وجود ندارد.

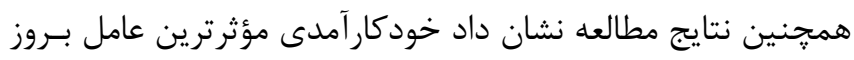

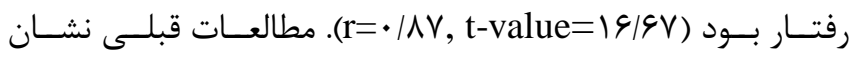

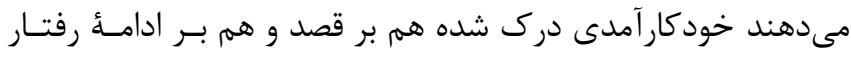

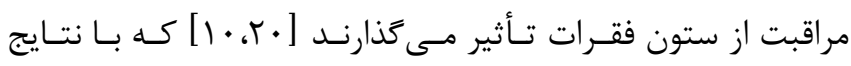

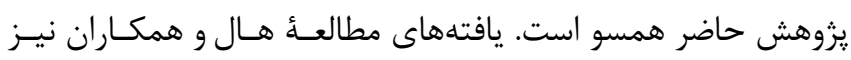

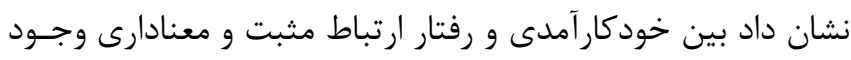

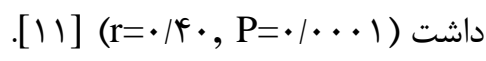

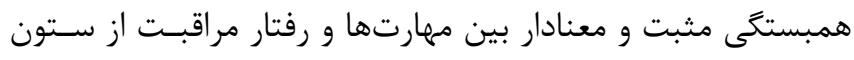

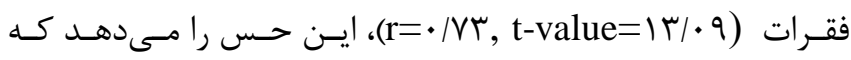

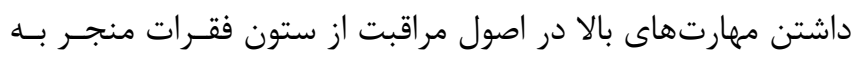

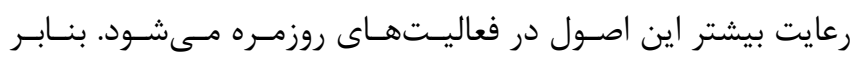

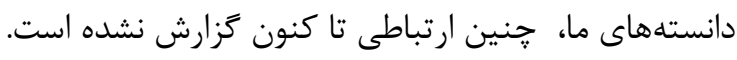

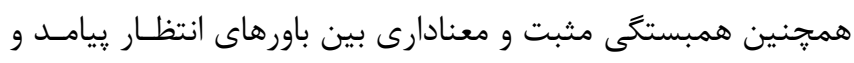

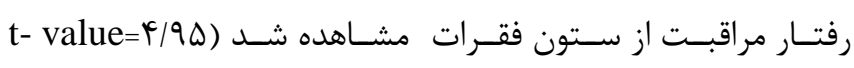
rr=•/DQ,

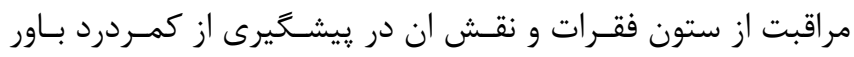

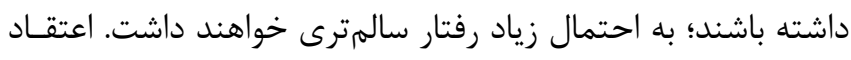

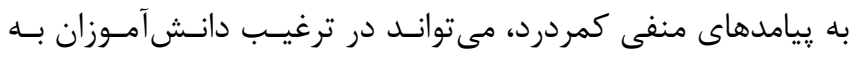

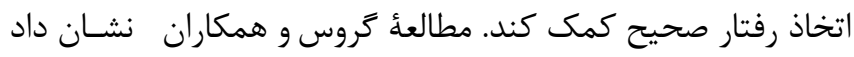

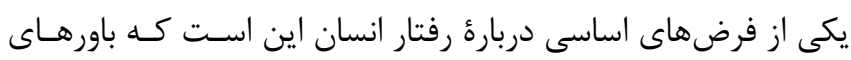

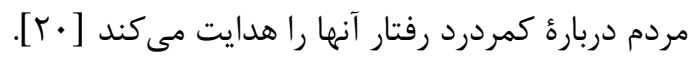




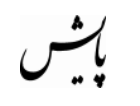

صديقه سادات طوافيان و همكاران

صديقهالسادات طوافيان: نظارت بر كل يزوهش و هـدايت آن، تأييـد نسخهُ نهايى

على منتظرى: مشاور يروزه، تحليل و تفسـير دادههـا، تأييـد نسـخهُ نهايى

\section{تشكر و قدردانى}

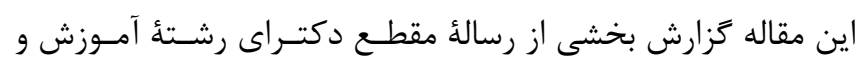

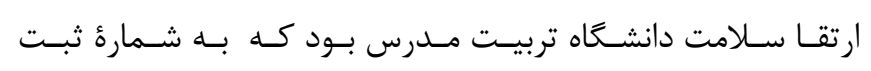

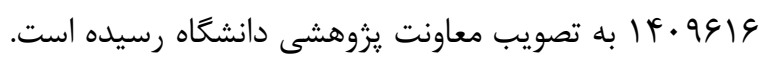

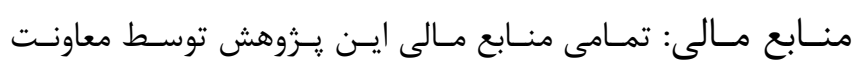

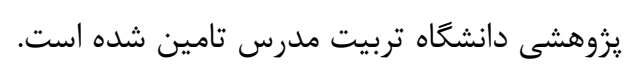

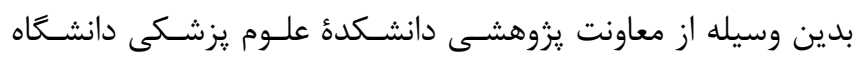

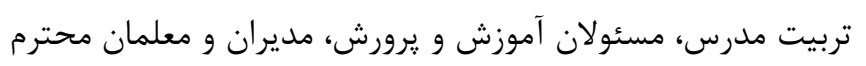

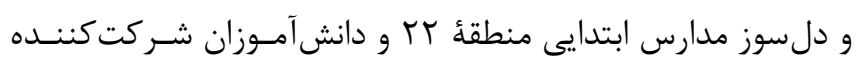
سياس ززارى مىنمايد.

\section{منابع}

1. Dullien S, Grifka J, Jansen P. Cluster-randomized, controlled evaluation of a teacher led multi factorial school-based back education program for 10 to 12 year old children. BMC Pediatric 2018;18:1-10

2. Amyra A, Ahmad A, Kamaruddin M, Nor S, Imanirwana $S$, Chin K. The association between backpack use and low back pain among pre-university students: A pilot study. J Taibah University Medical Science [Internet] 2018;13:205-9 Available from: https://doi.org/10.1016/j.jtumed.2017.06.

3. Kamper SJ, Henschke N, Hestbaek L, Dunn KM, Williams CM. Musculoskeletal pain in children and adolescents. Brazillian Journal of Physical Therapy 2016;20:275-84

4. Kamper SJ, Parma T, Williams CM. The prevalence, risk factors, prognosis and treatment for back pain in children and adolescents: An overview of systematic reviews. Best Practice \& Research Clinical Rheumatology [Internet] 2017;1-16 Available from: http://dx.doi.org/10.1016/j.berh.2017.04.003

5. Dianat I, Alipour A, Jafarabadi MA. Multigroup latent class model of musculoskeletal pain combinations in children / adolescents: identifying high-risk groups by gender and age. The Journal of Headache and Pain [Internet] 2018;1-8 Available from: http://dx.doi.org/10.1186/s10194-018-0880-0

6. Khanzada S, Khanzada S kanwal, Khan M sarfaraz, Shaikh S, Mirza R Ali, Naaz E, et al. Revalence of

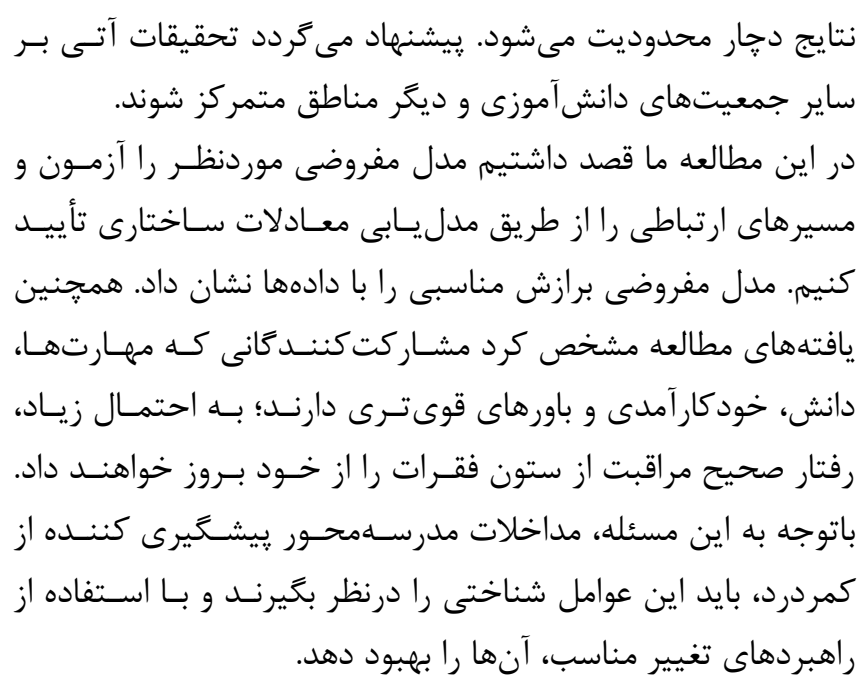

سهم نويسند

زهرا اكبرى جهرهبرق: يزوهشعر اصلى، جمع كنندهُ دادههـا، تحليـل

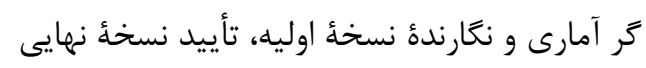

backache among school going children of hyderabad, sindh. International Journal of Physiotherapy 2016; 3:11-4

7. Maher C, Underwood M, Buchbinder R. Nonspecifi c low back pain. Lancet 2017; 389:736-47

8. Janakiraman B, Ravichandran H, Demeke S, Fasika S. Reported influences of backpack on postural deviation among school children: A systematic review. Journal of Education and Health Promotion 2017; 6:1-12

9. Dianat I, Alipour A, Jafarabadi MA, Traffic R. Prevalence and risk factors of low back pain among school age children in Iran. Health Promotion Perspectives 2017;7:223-9

10. Dolphens MBC, Danneels LDDC, Ilse De Bourdeaudhuij Greet Cardon. Longterm effectiveness of a back education programme in elementary schoolchildren: an 8-year follow-up study. European Spine Journal 2011; 20:2134-42

11. Hall E, Chai W, Koszewski W, Albrecht J. Development and validation of a social cognitive theory-based survey for elementary nutrition education program. International Journal of Behavioral Nutrition and Physical Activity 2015;12:1-12

12. Plotnikoff RC, Costigan SA, Karunamuni N, Lubans DR. Social cognitive theories used to e xplain physical activity behavior in adolescents: A systematic review and meta-analysis. Preventive Medicine (Baltim) [Internet] 2013;56:245-53 Available from: 
http://dx.doi.org/10.1016/j.ypmed.2013.01.013

13. Akbari-Chehrehbargh Z, Tavafian SS, Montazeri A. Effectiveness of a theory-based back care intervention on spine-related behavior among pupils: a school-based randomised controlled trial (T-Bak Study). BMC Public Health 2020; 20:805

14. Glanz K, Rimer BK, Viswanath K. Health Behavior and Health Education: Theory, Research and Practice. $4^{\text {th }}$ Edition. San Francisco: Jossey-Bass publisher, 2008

15. Kline, Rex B. Principles and practice of structural equation modeling. $3^{\text {st }}$ Edition. The Guilford Press: New York, London, 2011

16. Lee H, Kim J. A Structural Equation Model on Korean Adolescents â€TM Excessive Use of Smartphones. Asian Nursing Research (Korean Society Nursing Science) 2018;12:91-8 https://doi.org/10.1016/j.anr.2018.03.002

17. Akbari-Chehrehbargh Z, Tavafian SS, Montazeri A. The Back-care Behavior Assessment Questionnaire (BABAQ) for schoolchildren: development and psychometric evaluation. BMC Public Health 2020; 20:1283
18. Cardon G, De Clercq D, De Bourdeaudhuij I. Effects of back care education in elementary schoolchildren. Acta Pediatria 2000;89:1010-7

19. Heiser, Leigh S, Belcher D, Anshel M, Fuller D. Effects of a back-pain prevention education program on knowledge of proper back care among fifth grade elementary students. [PhD thesis] ProQuest Dissertations and Theses [Internet]; 2014 Retrieved from http://search.proquest.com/docview/1545616059 20. Gross DP, Deshpande S, Werner EL, Reneman MF, Miciak MA, Buchbinder R. Fostering change in back pain beliefs and behaviors: when public education is not enough. Spine Journal 2012;12:979-88

21. Santos NB Dos, Sedrez JA, Candotti CT, Vieira A. Immediate and Follow-Up Effects of a Posture Education Program for Elementary School Students. Review of Paulo Pediatria 2017;35:199-206

22. Noll M, Candotti C 'udia T^, Vieira A, Fagundes J. Back Pain and Body Posture Evaluation Instrument (BackPEI): development, content validation and reproducibility. International Journal of Public Health 2013;565-72 


\title{
ABSTRACT \\ The structural relationship between spine-related behavior among pupils and the constructs of social cognitive theory: A structural equation modeling analysis
}

\author{
Zahra Akbari-Chehrehbargh $^{1}$, Sedigheh Sadat Tavafian ${ }^{1 *}$, Ali Montazeri ${ }^{2}$ \\ 1. Faculty of Medical Sciences, Tarbiat Modares University, Tehran, Iran \\ 2. Health Metrics Research Center, Iranian Institute for Health Sciences Research, ACECR, Tehran, Iran
}

Payesh 2020; 19 (5): $581-589$

Accepted for publication: 26 August 2020

[EPub a head of print 28 September 2020]

Objective (s): Low back pain is a common disorder of spine. One of the most important concerns is the increasing rate of low back pain in schoolchildren that might predict adult disability. The purpose of this study was to explain the structural relationship between low back pain and spine-related behavior among pupils via structural equation modeling based on social cognitive theory (SCT). The hypothesized model included skills, knowledge of spine care principals, self-efficacy, and expectation beliefs as independent variables, and spine-related behavior as a dependent variable.

Methods: We collected data from 204 students attending public elementary schools using a structured questionnaire in Tehran, Iran from October 2018 to March 2019. The hypothesized model was examined via a structural equation modeling (SEM) analysis using SPSS, version 24.0, and LISREL, version 8.80.

Results: The effect of skills, knowledge, self-efficacy and beliefs on spine-related behavior was $0.73(t-v a l u e=13.09), 0.42(t-$ value=6.71), $0.87(\mathrm{t}$-value=16.67), and $0.55(\mathrm{t}$-value=4.95) respectively. In addition, various indicators, such as Comparative Fit Index (CFI), Normed Fit Index (NFI), $\chi 2 /$ df, and Root Mean Squared Error of Approximation (RMSEA) showed the good fitness of the models.

Conclusion: The results revealed that the pupils who had more confident, skills, expectation beliefs, and knowledge were more likely to perform proper spine-related behavior. In this regard, school-based low back pain prevention interventions should be addressed using key cognitive factors that consider the potential change strategies.

Key Words: Social Cognitive Theory, Spine-related behavior, Pupils, Structural equation modeling

\footnotetext{
${ }^{*}$ Corresponding author: Faculty of Medical Sciences, Tarbiat Modares University, Tehran, Iran

E-mail: tavafian@modares.ac.ir
} 